\title{
PARTES EXTRA PARTES. ÉTENDUE ET IMPÉNÉTRABILITÉ DANS LA CORRESPONDANCE ENTRE DESCARTES ET MORE
}

La correspondance entre Descartes et More peut être considérée comme un laboratoire, où sont débattues les principales questions de la philosophie naturelle et de la métaphysique du XvII ${ }^{\mathrm{e}}$ siècle : sur la présence de l'âme et de Dieu dans le monde, son immensité et ubiquité, sur la constitution corpusculaire de la matière, l'infinité du monde, sur l'union de l'âme et du corps, l'existence d'une âme des bêtes, ainsi que sur des questions plus spécifiques sur la physique et l'optique cartésiennes. Parmi ces questions, la discussion autour de la nature de l'étendue et de la res extensa, en particulier le problème de l'adéquation de la définition cartésienne du corps par l'étendue seule dont découlerait l'impénétrabilité, a depuis longtemps attiré l'attention des historiens de la philosophie ${ }^{1}$.

Toutefois l'attribution au corps et à l'étendue d'une structure partes extra partes joue un rôle crucial encore suffisamment exploré. Si être étendu implique d'avoir des parties distinctes liées par une relation d'extériorité, il s'ensuit que toute entité étendue possède une structure méréologiquement complexe. Or cette dimension méréologique est essentielle non seulement pour l'analyse des corps et de l'étendue corporelle, mais également pour le rapport des substances immatérielles à l'étendue. Ainsi, dans l'Enchiridion metaphysicum, Henry More invente le néologisme "holenmérisme " pour désigner les théories selon lesquelles l'âme est

* Abréviations utilisées : AT $=$ Euvres de Descartes, éd. par Ch. Adam et P. Tannery, nouv. présent. par J. Beaude, P. Costabel, A. Gabbey et B. Rochot, 11 vols., Paris, Vrin, 1964$1974 ; \mathrm{PP}=$ Descartes, Principia Philosophiae $; \mathrm{OO}=$ Henrici Mori Opera omnia, tum que latine, tum qua anglice scripta sunt; nunc vero latinitate donata, 3 vol., Londres, 1675-1679 (réimpr. anastatique : Hildesheim, G. Olms, 1966 ; EM = Henry More, Enchiridion metaphysicum (1671); DM = Francisco Suárez, Disputationes metaphysicae (1597), in Opera omnia, éd. C. Berton, Paris, Vivès, 1856, 26 vol. vol. 25 et 26.

1. La correspondance a fait l'objet sur ces questions en général de nombreuses études classiques. Parmi celles-ci, on mentionnera Alexandre Koyré, Du monde clos à l'univers infini, Paris, Gallimard, 1962; Edward Grant, Much Ado about Nothing, Theories of Space and Vacuum from the Middle Ages to the Scientific Revolution, Cambridge, Cambridge University Press, 1981. L'article de Claire Schwartz dans ce numéro abordent aussi ces questions. 
localisée dans le corps tota in toto et tota in qualibet parte ${ }^{2}$. Après Hobbes, il attribue cette thèse aux scolastiques ${ }^{3}$. Les substances immatérielles ne sont pas étendues par parties distinctes, mais occupent entièrement plusieurs lieux à la fois. On peut comprendre le holenmérisme comme une forme de multi-localisation, au sens où les universaux dans une théorie réaliste sont entièrement présents aux lieux où ils sont instanciés. Les universaux ne sont pas pour autant étendus spatialement. Il est néanmoins possible d'envisager une hypothétique entité méréologiquement simple et étendue spatialement. Dans la philosophie contemporaine, ces entités hypothétiques sont appelées des étendus simples ${ }^{4}$. L'introduction dans l'ontologie d'entités multilocalisées ou d'étendus simples implique de renoncer à ce que l'on peut appeler un principe d'harmonie méréologique, c'est-à-dire au principe selon lequel une entité spatialement étendue est méréologiquement complexe et réciproquement une entité simple ne peut occuper un lieu méréologiquement complexe, donc une étendue partes extra partes 5 . De ce point de vue la question de l'impénétrabilité peut être envisagée comme un problème relevant d'une théorie de la localisation : deux entités se pénètrent si elles occupent un même lieu sans que cela affecte la nature de l'une ou l'autre.

Dans ce qui suit, on s'efforcera de montrer comment la discussion entre Descartes et More sur l'adéquation de la définition des corps repose sur deux compréhensions différentes chez les deux penseurs de ce qu'implique le fait d'être étendu et en particulier le fait d'exister partes extra partes. On remontera dans un deuxième temps aux discussions médiévales sur la quantité d'où découlent les positions opposées sur le rapport entre étendue et impénétrabilité. Cela permettra dans la troisième partie de revenir sur l'argument cartésien de la nécessaire impénétrabilité de toute res extensa. Enfin, dans la dernière partie, on montrera de quelle façon les aspects méréologiques de la discussion sur l'étendue permettent de mieux comprendre la nature de deux propriétés attribuées par More à l'étendue spirituelle dans ses textes ultérieurs, à savoir la spissitude et l'indiscerpibilité.

2. EM, chap. XXVII, $₫ 1$, OO II-1, 307. Voir l'article de David Leech dans ce numéro ainsi que Igor Agostini, "Henry More e le fonti della dottrina dell' estensione spirituale ", in P. Dessì et B. Lotti (ed.), Eredità cartesiane nella cultura britannica, Florence, Le Lettere, 2011, pp. 49-69 ; Igor Agostini, "Henry More e l'olenmerismo ", Nouvelles de la République des Lettres, 2006, pp. 7-23 ; Jasper Reid, The Metaphysics of Henry More, Dordrecht, Springer, 2012, pp. 158-174. p. 686.

3. Thomas Hobbes, Léviathan, chap. XLVI, $\$ 19$, trad. F. Tricaud, Paris, Sirey, 1971,

4. Un étendu simple est une entité étendue et dépourvue de parties propres, autrement dit méréologiquement simple. Cf. Ned Markosian, "Simples ", Australasian Journal of Philosophy 76 (1998), pp. 213-226.

5. Pour un aperçu des discussions contemporaines sur les "théories de la localisation ", voir Cody Gilmore "Location and Mereology", The Stanford Encyclopedia of Philosophy (Summer 2013 Edition), Edward N. Zalta (ed.), URL $=<$ http://plato.stanford.edu/archives/ sum2013/entries/location-mereology/> 2013. 


\section{La définition de l'étendue chez Descartes et sa critique par More}

Les problèmes relatifs à la définition de l'étendue et des corps et au rapport des âmes et de Dieu à l'espace et au lieu occupent une place centrale dans la correspondance ${ }^{6}$. Ces deux problèmes n'en font qu'un aux yeux de Henry More. Ce dernier ouvre ainsi la correspondance par une critique de la définition cartésienne du corps comme res extensa.

Descartes définit comme on sait le corps comme une chose étendue tridimensionnelle (res extensa in longum, latum et profundum, AT VIII-1, 42). Or, entre la substance et son attribut principal, la distinction est seulement une distinction de raison. Autrement dit le corps n'est pas l'étendue d'une chose, au sens où l'étendue serait distincte de cette chose, mais est l'étendue elle-même ${ }^{7}$, thèse qui figure déjà dans la règle XIV des Regulae (AT X, 442-43). Au chapitre VI du Monde, Descartes oppose la conception de la matière qu'il imagine aux théories scolastiques qui distinguent la matière des corps de leur quantité et de leur étendue :

[...] Ils [i.e. les Philosophes] la [i.e. la matière] veulent distinguer de sa propre quantité et de son étendue extérieure, c'est-à-dire de la propriété qu'elle a d'occuper l'espace [...] La quantité de la matière que j'ai décrite ne diffère non plus de sa substance que le nombre fait des choses nombrées, et [...] je conçois son étendue ou la propriété qu'elle a d'occuper de l'espace non point comme un accident, mais comme sa vraie forme et son essence (AT XI, 35-36).

Le rapport de l'étendue à la chose étendue ou corps est celui du nombre à la chose nombrée. Dans les Principes, Descartes recourt à la même comparaison pour rendre compte de la différence entre l'espace ou lieu interne et la res extensa ${ }^{8}$.

La radicalité de la thèse cartésienne consiste dans sa triple identification du corps, de l'étendue et de l'espace. Elle implique une série de conséquences examinées dans les Principes de la philosophie. D'abord, l'identification du corps à l'étendue implique le refus de la condensation et de la raréfaction absolues (PP II, 5-8).

6. Voir Daniel Garber, Descartes's Metaphysical Physics, Chicago, The University of Chicago Press, 1992, en particulier pp. 145-148; Robert Pasnau, "Mind and Extension (Descartes, Hobbes, More) ", in H. Lagerlund (ed.), Forming the Mind. Essays on the Internal Senses and the Mind/Body Problem from Avicenna to the Medical Enlightenment, Springer, 2007, pp. 283-310 ; Robert Pasnau, Metaphysical Themes 1274-1671, Oxford, Clarendon Press, 2011 ; Jasper Reid, op. cit. ; Emanuela Scribano, Da Descartes a Spinoza. Percorsi della teologia razionale nel Seicento, Milan, Franco Angeli, 1988 ; Sur le problème de la localisation de l'âme et de l'union avec le corps, voir aussi Dennis Des Chene, Lifés Form: Late Aristotelian Conceptions of the Soul, Ithaca, Cornell UP, 2000, chap. X; Marleen Rozemond, "Descartes, Mind-Body Union, and Holenmerism ", Philosophical Topics, 2003, 31, pp. 343367 ; Jasper Reid, "The Spatial Presence of Spirits among Cartesians ", The Journal of the History of Philosophy, 2008, 46, pp. 91-118.

7. Calvin G. Normore, "Descartes and the Metaphysics of Extension", in J. Broughton et J. Carriero (eds.), A Companion to Descartes, Oxford, Blackwell, 2008, pp. 271-287, insiste sur ce point à la p. 272 .

8. PP II, 8, AT VIII-1 44. 
Il est impossible qu'un corps occupe un espace plus ou moins grand étant donné son identité à l'étendue qu'il occupe. En second lieu, l'identification de l'étendue corporelle à l'espace implique l'impossibilité absolue du vide (PP II, 9-19).

Henry More conteste une telle identification et appuie ce refus sur une critique de la définition du corps par l'étendue, alors même qu'il admet, dans l'explication des phénomènes naturels, l'explication cartésienne de la condensation et raréfaction ainsi que l'inexistence d'un vide actuel' ${ }^{9}$. C'est ainsi sur le plan métaphysique que se situe l'enjeu de la discussion.

L’objection de More contre la définition cartésienne des corps par la seule étendue est bien connue : elle est plus large que le défini dans la mesure où les substances incorporelles sont elles aussi étendues. Étant une propriété commune aux corps et aux esprits, l'étendue ne peut constituer à elle seule l'essence des corps. Il propose en retour de définir le corps comme une substance tangible et impénétrable (AT V, 240) ${ }^{10}$. La discussion s'engage alors sur deux fronts qui se poursuivent de lettre en lettre en se recoupant régulièrement. Le premier front relève de l'analyse du concept d'extensio, tandis que le second engage la question de l'étendue des esprits : ceux-ci sont-ils étendus, occupent-ils un lieu et, le cas échéant, en quel sens ?

Descartes refuse de reconnaitre le caractère inadéquat de sa définition du corps et à cet effet il s'appuie sur la distinction de la logique traditionnelle entre un attribut par soi et l'un des types d'accidents distingués par Porphyre dans l'Isagogê, le proprium quarto modo, comme le rire ${ }^{11}$. L'impénétrabilité est un accident de ce genre puisqu'elle convient à tous les corps sans constituer l'essence du corps, dans la mesure où le concept d'impénétrabilité présuppose celui d'étendue qui, étant une notion primitive, se conçoit d'elle-même (PP I, 53, AT VIII-1, 25). L'argument par lequel il établit l'antériorité conceptuelle de l'étendue dans la définition du corps joue sur l'opposition entre le caractère absolu de la notion d'étendue et la nature relative de l'impénétrabilité :

La tangibilité et l'impénétrabilité ont une relation aux parties et présupposent le concept de division ou de terme. Or nous pouvons concevoir un corps continu d'une grandeur indéterminée, ou indéfini, dans lequel on ne considère rien en dehors de l'étendue (Descartes à More, 5 février 1649, AT V, 269 ${ }^{12}$ ).

Descartes argumente de la manière suivante : l'impénétrabilité est un concept relationnel. En effet est impénétrable ce qui est tel que deux de ses parties

9. ATV, 240-241 pour le vide ; ATV, 241 pour la condensation et la raréfaction.

10. More ouvre la voie à une critique de la conception cartésienne faisant de l'étendue l'essence des corps qui sera reprise tant par Locke (II, xiii, \$ 11) que par Leibniz (Remarques sur la partie générale des principes de Descartes, GP IV, 364-65, trad. P. Schrecker, in Leibniz, Opuscules philosophiques choisis, Paris, Vrin, 2001², p. 67)

11. Le propre du quatrième type désigne une propriété qui convient universellement à un terme commun et ne convient qu’à lui et qui lui convient nécessairement sans constituer son essence. Voir Guillaume d'Ockham, Somme de logique, I, chap. XXIV, trad. J. Biard, Mauvezin, T.E.R., 1993, pp. 83-84.

12. Nous traduisons, ainsi que les autres citations extraites de la correspondance. 
ne peuvent être co-localisées. Le concept d'impénétrabilité présuppose donc une distinction de parties de grandeurs déterminées. Or, poursuit Descartes, il est possible à l'inverse de concevoir un corps continu d'une grandeur et figure indéterminée sans concevoir de parties distinctes dans ce corps. Donc l'attribut par lequel se définit le corps en général, c'est l'étendue simple ${ }^{13}$. La conclusion de cet argument signifie que le concept d'étendue n'implique pas la possession d'une structure méréologique complexe, autrement dit on peut concevoir une étendue indéfinie véritable, sans considérer de parties distinctes en elle. La conséquence indirecte est que la notion d'impénétrabilité ne peut s'appliquer à ce corps étendu indéterminé14 .

Or il y a là un écart et une incohérence potentielle avec ce qu'écrit Descartes un peu plus loin dans la même lettre :

En effet, par un être étendu chacun conçoit communément quelque chose d'imaginable (qu'il s'agisse d'un être de raison ou d'un être réel, je laisse cela en suspens ici), et on peut par l'imagination distinguer dans cet être diverses parties de grandeur et figure déterminées, dont l'une n'est point l'autre, et on peut aussi transporter par l'imagination l'une dans le lieu des autres, mais on ne peut en imaginer deux à la fois en un seul et même lieu (AT V, 270).

Descartes définit l'ens extensum comme une entité (i) imaginable, (ii) pourvue de parties distinctes ayant grandeur et figure déterminée ; (iii) ces parties étant mobiles et (iv) néanmoins telles que deux d'entre elles ne peuvent occuper le même lieu. Le caractère imaginable de l'étendue et le caractère mobile de ses parties sont secondaires pour notre propos. Le point décisif est que le concept d'être étendu inclut la division en parties actuelles distinctes ainsi que celui d'impénétrabilité ${ }^{15}$. Il y a là en effet une contradiction apparente avec la thèse précédente. On ne saurait la résoudre en rapportant la première à l'étendue considérée en faisant abstraction du corps, ce que Descartes nomme l'extensio in genere dans les Principes, qui n'est qu'un modus cogitandi dépourvu de réalité propre hors de l'esprit ${ }^{16}$. En effet, dans le premier passage, Descartes parle bien d'un corps qui est conçu comme indéfini sans considération de parties et non d'un espace abstrait.

Il y a là une véritable tension, révélatrice de la dualité de point de vue sur l'étendue chez Descartes, qui peut être considérée tantôt en quelque sorte "de haut en bas ", tantôt à l'inverse "de bas en haut". Le premier point de vue correspond à celui de l'étendue comme notion primitive. Dans ce cas, l'identification de parties pourvues de relations d'extériorité est secondaire. Le second point de vue correspond précisément à l'étendue envisagée à partir

13. Il y a un écart ici avec la formulation de la Méditation cinquième où Descartes explique qu'il imagine distinctement une étendue tridimensionnelle dans laquelle il distingue diverses parties auxquelles il assigne grandeur, figure, situs et mouvement déterminés (AT VII, 63).

14. C'est ce que remarque Daniel Garber, op. cit., p. 148.

15. Voir aussi Sixièmes réponses, AT VII, 442 : vera enim corporis extensio talis est, ut omnem partium penetrabilitatem excludat.

16. Respectivement PP II, 12, AT VIII-1, 46 et I, 58, AT VIII-1, 27. 
de ses conditions ontologiques. De ce point de vue la divisibilité en parties distinctes est première et par conséquent l'idée d'une pluralité de parties liées par la relation d'extériorité l'est également. Le problème est que Descartes fait jouer successivement ces deux points de vue sur l'étendue pour répondre à l'objection de More concernant sa définition de l'étendue. D'une part en effet, l'analyse de l'étendue fait apparaitre l'impénétrabilité comme une de ses propriétés définitionnelles. D'autre part elle lui est antérieure, en ce qu'elle est la notion primitive sous laquelle tombent les corps. La question qui se pose alors est de savoir s'il est possible de fonder l'impénétrabilité dans l'une des autres propriétés énoncées, imaginabilité, extériorité et divisibilité des parties distinctes. L'imaginabilité ne joue pas de rôle direct dans la mesure où elle est une propriété relative à notre connaissance. Il reste alors la divisibilité, la mobilité et le fait que l'étendue soit partes extra partes. Les deux premières sont subordonnées à la dernière qui est la condition fondamentale de l'impénétrabilité. L'argument décisif de Descartes contre l'objection de More consiste ainsi à montrer que l'impénétrabilité découle de l'extériorité des parties.

L'ambiguïté des deux points de vue sur l'extensio et la res extensa apparaît nettement dans la suite de la correspondance. Dans sa lettre du 5 mars 1649, More note que le concept d'extensio est relationnel :

Quand vous définissez vous-même le corps une chose étendue, je remarque avec cela que cette même étendue consiste dans un rapport des parties entre elles, en tant qu'elles sont produites les unes en dehors des autres (aliae extra alias) et il est manifeste que ce rapport n'est pas une chose absolue (rem absolutam) (AT V, 299-300).

L'étendue impliquant une relation d'extériorité des parties entre elles, elle n'est pas une res absoluta et ne permet donc pas de définir l'essence des corps qui exige une propriété plus fondamentale ${ }^{17}$. Descartes répond dans sa lettre du 15 avril que l'on peut concevoir l'étendue sans penser à la relation des parties entre elles:

Mais bien que dans l'étendue nous comprenions facilement le rapport des parties entre elles, il me paraît cependant que je perçois très bien l'étendue, quoique je ne pense pas du tout au rapport des parties entres elles; ce que vous devez admettre à plus forte raison que moi, parce que vous concevez l'étendue de telle façon qu'elle convienne à Dieu et que vous n'admettez pourtant aucune partie en lui (AT V, $341)$.

La première partie de la réponse indique clairement que c'est du point de vue de la pensée que la notion d'étendue peut être conçue sans la relation entre ses parties distinctes. Cette distinction est plus qu'une distinction de raison puisque Descartes indique "percevoir très bien" (optime percipere) cette

17. De manière surprenante, cette objection anticipe celle que formule régulièrement Leibniz à l'encontre de la définition cartésienne des corps. Voir les Animadversiones de 1692, GP IV, 364-365, op. cit., p. 67. 
étendue sans la relation entre des parties. Il ne s'agit pas non plus d'une distinction réelle dans la mesure où réciproquement l'on ne peut percevoir clairement et distinctement une relation d'extériorité entre parties sans l'étendue absolue. Il reste alors la distinction modale qui paraît correspondre à ce cas de figure. Mais celle-ci ne va pas sans poser problème, puisque si les parties de l'étendue sont distinguées modalement de l'étendue elle-même, elles devraient l'être également entre elles ${ }^{18}$. Or les parties de l'étendue sont réellement distinctes entre elles et ne sont donc pas de simples modes de la res extensa ${ }^{19}$. Il s'agit là d'une question difficile. La difficulté tient à ce que si la séparabilité d'existence est l'unique critère de distinction réelle et l'indépendance ontologique celui de la substantialité, alors la res extensa elle-même ne serait pas une substance - puisqu'elle dépend de celle de ses parties. Inversement, si l'on part de l'immutabilité de la res extensa, alors nulle de ses parties n'est ontologiquement indépendante et par conséquent elle ne serait pas une substance. Le problème est ainsi que le choix entre la distinction réelle ou modale engage directement la question de l'individuation des substances corporelles dont il n'est pas directement question dans l'échange avec More. En d'autres termes, la tension réside dans le fait que l'étendue est épistémiquement un concept simple alors qu'elle est complexe au plan ontologique.

Dans la dernière phrase du passage précédent, Descartes retourne l'objection de More contre celui-ci. Une preuve d'après lui que le philosophe anglais peut concevoir l'étendue sans penser au rapport des parties est qu'il reconnaît une étendue divine. Or Dieu est simple. Donc on peut concevoir une étendue dépourvue de parties. Si l'on néglige le caractère purement ad hominem de l'argument, on pensera que l'étendue cartésienne est non seulement épistémiquement mais également ontologiquement et méréologiquement simple, ce qu'elle n'est évidemment pas (cf. AT V, 272).

Mais, en retour, More juge-t-il en effet que l'étendue divine est dépourvue de parties et que Dieu est un "étendu simple " ? La réponse à cette question est moins simple qu'il n'y parait. Dans sa lettre du 5 mars, il donne en effet un certain nombre d'éléments de réponse, mais ces éléments ne vont pas tous dans le même sens.

D'un côté, il affirme clairement que la notion d'étendue implique celle d'extériorité des parties entre elles, au point d'en faire une quasi-définition: "Extendi nihil aliud innuit nisi partes extare extra partes" (AT V, 307). Dans une lettre à Anne Conway de 1651, il définit semblablement l'étendue $^{20}$. Le contexte de cette assertion est identique dans les deux lettres. More

18. Voir PP I, 61, AT VIII-1, 29 : « Unum quidem modum absque alio possimus agnoscere ; sed neutrum [i.e. deux modes d'une même substance] tamen sine eadem substantia cui insunt. "

19. PP I, 60, AT VIII-1, 28 ; Lettre à Gibieuf, 19 janvier 1642, AT III, 477.

20. Henry More à Anne Conway, 5 mai [1651], éd. Alan Gabbey, «Anne Conway et Henry More. Lettres sur Descartes (1650-1651) ", Archives de philosophie 40 (1977), pp. 379404, pp. 387-88: «Distance or Extension, in its very nature implies no more than this, to have partem extra partem, that is, to have explicated parts. " 
cherche à établir le caractère "super-transcendantal » de l'étendue et de la durée, qui s'appliquent aux êtres comme aux non-êtres (AT V, 306), ce qui implique la possibilité d'un vide absolu (AT V, 302). Dans ses écrits ultérieurs, il y renonce et fait de l'étendue un attribut de l'ens quatenus ens ${ }^{21}$. Cela ne l'empêche pas cependant de continuer à définir l'étendue à partir de la notion de parties : "It being the very essence of whatsoever is, to have Parts or Extension in some measure or other ${ }^{22}$."

D'un autre côté cependant, More a affirmé un peu auparavant dans la même lettre l'existence d'une alia vera extensio que l'étendue corporelle. De cette étendue, il est dit qu'elle exclut l'impénétrabilité, mais s'accompagne d'autres propriétés : elle est figurée et variable (AT V, 301). Un peu plus loin, à propos de l'omniprésence divine, More soutient qu'elle est indivisible, bien qu'elle soit une étendue véritable car elle est mesurable ( $c f$. AT V, 302). Il présente alors la modalité de cette omniprésence dans un vocabulaire holenmérien :

En outre, puisque Dieu, pour autant que l'esprit humain saisit Dieu, est tout entier partout, et qu'il est présent par son essence entière à tous les lieux ou espaces et à tous les points de ces espaces, il ne s'ensuit pas qu'il ait des parties extérieures les unes aux autres (quod partes haberet extra partes), ou, ce qui est en est une conséquence, qu'il soit divisible, quoiqu'il occupe étroitement et de manière serrée tous les lieux, sans laisser aucun intervalle. Ainsi je reconnais que la présence divine, ou son amplitude, comme vous l'appelez vous-même, est mesurable, mais en aucune façon que Dieu lui-même soit divisible (AT V, 305).

L’omniprésence divine implique une forme d'étendue que More appelle aussi son amplitude. Dieu est parfaitement simple et indivisible, ce qui suppose qu'il n'ait pas une structure partes extra partes. More use d'un vocabulaire holenmérien, confirmé dans la suite de la lettre lorsqu'il évoque la réduplication infinie du centre divin dans l'espace tout entier (AT V, 305-6). Et il oppose cette forme d'étendue à l'étendue corporelle :

[L'étendue incréée] naît de la répétition ubiquitaire de l'essence divine tout entière, celle-ci [l'étendue créée] de l'application extérieure, mais immédiate des parties et de leur juxtaposition (AT V, 308).

Or la structure méréologiquement complexe de l'étendue créée s’accompagne de son impénétrabilité, par contraste avec l'étendue divine qui est pénétrable. Ceci ne laisse pas de poser problème, puisque More semble donc, comme Descartes, souscrire au principe d'implication de la complexité méréologique à l'impossibilité de la co-localisation, autrement dit à l'impénétrabilité. À ce

21. Voir Divine Dialogues I, $\$ 5$, cité in J. Reid, op. cit., p. 185 : « Extension or Amplitude is an intrinsecal or essential Property of Ens quatenus Ens, as the Metaphysicians phrase it "; $E M$, chap. II, $\$ 8$, OO II-1, 144 .

22. The Immortality of the Soul, The Preface $\$ 3$, in Henry More, A Collection of Several Philosophical Writings, Londres, 1662, p. 3 
compte, la discussion sur l'inadéquation de la définition cartésienne serait purement verbale. Il demeure néanmoins le fait que More continue à parler d'étendue véritable pour caractériser l'omniprésence divine. Il appuie cette affirmation sur le fait que la présence de Dieu est mesurable, autrement dit parce qu'elle occupe un espace tridimensionnel infini ${ }^{23}$. De même, les esprits créés sont eux-mêmes étendus parce qu'ils sont non seulement mesurables, mais encore figurés - un esprit occupe une étendue d'espace coïncidant avec le corps qu'il anime - et variable - un esprit occupe une étendue d'espace plus ou moins grande. More paraît bien admettre dans la correspondance des entités multi-localisées et même des étendus simples. Aussi l'argument ad hominem de Descartes n'est-il pas dénué de fondement. Néanmoins, on comprend mal pour quelle raison More aurait soutenu dans la même lettre que la structure partes extra partes constituait l'essence de l'étendue en général s'il en exemptait l'étendue ou l'amplitude des substances spirituelles et de Dieu.

Afin de résoudre cette difficulté, il est nécessaire de revenir à la manière dont More répond à l'analyse cartésienne de l'espace identifié à l'ens extensum. Descartes soutient que cet espace contient des parties de grandeurs et figures variées, mobiles et impénétrables (AT V, 271). La réponse de More nous éclaire sur le rapport des parties à la divisibilité et à l'impénétrabilité :

Mon imagination n'en est certes pas capable, non plus que de concevoir, si on les transporte, que les parties d'un espace vide n'absorbent pas les autres, ne coïncident tout à fait et ne se pénètrent mutuellement (AT V, 302).

Les parties de l'espace sont ainsi immuables. Mais dans l'hypothèse impossible où elles le seraient, une portion d'étendue spatiale pourrait coïncider avec l'autre, de telle façon qu'elles seraient parfaitement co-localisées. Nous reviendrons plus loin sur l'argument développé par Descartes dans sa lettre du 15 avril. On retiendra ici que la possession d'une structure méréologique complexe, partes extra partes dans le cas de l'espace n'entrâne pas l'impénétrabilité.

Si l'on admet que les questions de More reflètent une position philosophique cohérente, on peut conclure provisoirement que, dès l'époque de ses lettres à Descartes, celui-ci ne distingue pas seulement deux, mais trois formes d'étendue :

1) L'étendue corporelle, qui possède une structure méréologiquement complexe, et qui est impénétrable.

2) L'étendue spatiale, qui possède une structure méréologiquement complexe et qui est pénétrable.

3) L'étendue spirituelle qui est méréologiquement simple et pénétrable. Celle-ci se distingue à son tour entre (i) une étendue spirituelle

23. Chez certains scolastiques comme Suárez, l'omniprésence divine s'accompagne inéluctablement d'une représentation d'une étendue spatiale dimensionnelle; cf. DM, disp. XXX, s. 7, n. 49-50. La nouveauté de More consiste à ériger en propriété ontologique ce qui chez Suárez est lié à une modalité de la représentation. 
incréée, qui est infinie, immuable et mesurable et (ii) une étendue spirituelle créée, finie, mesurable, figurée et variable.

Il est nécessaire de garder présente à l'esprit cette typologie et en particulier la distinction entre (2) et (3) que les écrits ultérieurs de More tendent à effacer, en raison de l'identification progressive de l'espace à l'étendue divine, achevée dans l'Enchiridion metaphysicum ${ }^{24}$. Il reste à éclaircir la raison pour laquelle la possession d'une structure méréologique complexe s'accompagne d'impénétrabilité dans le cas des corps, alors qu'elle s'accompagne de pénétrabilité avec l'étendue spatiale. Descartes soutient que l'étendue, ou plus précisément le fait d'exister partes extra partes implique l'impénétrabilité. More semble à l'inverse soutenir une position ambivalente. Cependant la réalité de l'espace, reconnue dès l'époque de la correspondance témoigne du fait que la complexité méréologique n'entraîne pas l'impénétrabilité. La discussion autour de l'impénétrabilité comporte donc bien une dimension méréologique centrale. Sous cet aspect elle prolonge les discussions médiévales sur le rapport entre matière, étendue et structure méréologique. C'est pourquoi, avant d'aller plus loin dans l'analyse de l'opposition entre Descartes et More, il convient de rappeler quelques-unes des positions qui émergent à l'occasion de ces discussions.

\section{2. Étendue et impénétrabilité au prisme des discussions scolastiques sur la quantité}

Létude de la nature et des propriétés de la catégorie de la quantité constitue le principal lieu de ces discussions chez les médiévaux. Le débat met aux prises le réalisme de la quantité à la thèse nominaliste ${ }^{25}$.

La théorie d'Ockham représente la position nominaliste la plus radicale sur la quantité. Celui-ci affirme que toute substance possèdant des parties intégrales ${ }^{26}$, autrement dit une structure méréologique complexe, est étendue et que toute étendue est nécessairement une grandeur, un quantum divisible à l'infini ${ }^{27}$. Être étendu ne se distingue pas du fait de posséder une

24. EM, chap. VIII, $\$ 8$, OO II-1, 167 ; Divine Dialogues, chap. III, $\$ 40 ; c f$. Jasper Reid, op. cit., p. 121 et pp. 212-215.

25. Sur cette question, on consultera essentiellement Anneliese Maier, "Das Problem der Quantität oder der Raümlichen Ausdehnung ", in Metaphysiche Anfangsgründe der Spätscholastischen Naturphilosophie, Rome, 1955, 141-223 ; Joël Biard, « La conception cartésienne de l'étendue et les débats médiévaux sur la quantité ", in Joël Biard et Roshdi Rashed, Descartes et le Moyen Âge, Paris, Vrin, 1997, pp. 349-361 ; Robert Pasnau, op. cit., chap. IV, XIV et XV.

26. Une partie intégrale est une partie quantitative, par ex. les branches et les racines sont des parties intégrales de l'arbre. Les scolastiques les distinguent des parties essentielles, que sont la forme et la matière ou le genre et la différence. Voir Thomas d'Aquin, Summa theologiae, I, q. 8, a. 2, ad 3 et Aristote, Métaphysique V, 25, 1023b12-25.

27. cf. Tractatus de corpore christi, chap. XVII, Opera Theologica (= OTh), vol. X, Saint Bonaventure, NY, 1986, p. 125. 
grandeur, autrement dit, c'est une étendue dimensionnelle appartenant intrinsèquement à la matière comme aux corps, composés de matière, formes et qualités ${ }^{28}$.

Le principal argument d'Ockham à l'appui de l'identification de la substance matérielle à un quantum étendu est un argument d'économie, dans lequel le recours à la potentia Dei absoluta joue le rôle d'une prémisse essentielle ${ }^{29}$. Dieu peut conserver inchangée une chose en détruisant tout ce qui est natura posterius, autrement dit ce dont elle est ontologiquement indépendante. Or, dans l'hypothèse où la quantité serait un accident distinct de la substance, Dieu pourrait supprimer celle-ci sans produire de changement dans la substance matérielle. En particulier, la matière, qui est essentiellement composée de parties, serait maintenue telle qu'elle était, c'est-à-dire en conservant les mêmes relations de distance entre ses parties. Or la quantité ne consiste précisément que dans les rapports de distance spatiale entre des parties ${ }^{30}$. Donc il est redondant de poser la quantité comme un accident réellement distinct de la substance. Le noyau du raisonnement consiste dans l'impossibilité absolue de modifier l'étendue d'une substance sans modifier la substance elle-même, soit en produisant un changement intrinsèque, par la suppression d'une des parties de la substance, soit en produisant un changement dans les rapports de situation des parties elles-mêmes. Ainsi l'étendue est inséparable de la matière.

L'identification de l'étendue au corps et à la matière entraine un certain nombre conséquences sur ses propriétés et sur le rapport entre méréologie et localisation qu'Ockham a nettement formulées dans la Summula philosophiae naturalis :

[1] Il est impossible qu'il y ait de la matière sans étendue. En effet [2] il n'est pas possible qu'il y ait de la matière s'il n'y a pas une partie distante d'une autre. D'où vient que, bien que les parties de la matière puissent être unies à la façon dont les parties de l'eau et de l'air peuvent l'être, néanmoins [3] les parties de la matière ne peuvent jamais être dans le même lieu [in eodem loco]. Et ainsi la matière possède toujours une partie distante d'une autre, et c'est en cela que consiste le fait que la matière soit étendue et quantifiée ou dimensionnée [et hoc est materiam esse extensam et quantam vel dimensionatam] (Summula I, chap. XIII, Opera philosophica, vol. VI, Saint Bonaventure, NY, 1984, p. 191, nous numérotons).

28. Comme le note justement Anneliese Maier art. cit., p. 196, c'est la conjonction de ces deux thèses qui démarque la position d'Ockham, d'autres auteurs (infra) ayant pu défendre auparavant l'idée que la matière possède une "quasi-étendue » ou diffusion dépourvue de structure corpusculaire ou méréologique.

29. L'argument est repris dans tous les écrits d'Ockham abordant cette question. Il fait l'objet d'un long développement dans le Tractatus de corpore Christi, chap. XII-XV, OTh X, 112-121; cf. Quodlibeta septem IV, q. 22-24 (OTh IX, pp. 403-415). Il est présenté plus succinctement dans la Somme de logique I, chap. XLIV, trad. cit., pp. 141-42.

30. Somme de logique I, chap. XLIV, p. 144 : «Quantitas continua permanens nihil aliud est nisi res una habens partem situaliter distantem a parte [...]. Et ideo, cum substantia habeat partem situaliter distantem a parte, et similiter qualitas, aliqua quantitas non erit alia res a substantia, et aliqua quantitas non erit alia res a quantitate. » 
Ce texte lie entre elles trois thèses définissant la position d'Ockham :

1. La matière est nécessairement étendue.

2. L'étendue elle-même consiste dans le fait d'être partes extra partes.

3. Tout ce qui est étendu est impénétrable.

Le rapport logique entre ces trois thèses explique la conception ockhamiste de l'étendue. (2) est la thèse fondamentale. Elle consiste d'abord dans l'affirmation du caractère méréologiquement complexe de toute étendue, donc des corps. L'étendue de la matière (1) en est une conséquence. Ainsi la notion d'étendue comme extension spatiale, n'est pas une notion primitive. Elle peut s'analyser à partir de la donnée d'une pluralité de parties et de relations spatiales entre celles-ci. Toutefois (1) n'est pas une conséquence immédiate de (2), mais de (2) et (3). En (2), Ockham affirme à la fois :

$(2$ ') tout ce qui est étendu possède une structure méréologiquement complexe (partes)

et

(2") les parties de l'étendue sont extérieures les unes autres (partes extra partes).

Bien que logiquement distinctes, Ockham tient pour équivalentes ces deux affirmations. Le passage de (2) à (3) est ce qui permet de justifier l'équivalence de (2') et (2"). Ce faisant, Ockham reprend une thèse admise depuis Aristote. Dans la Physique ce dernier rejette la thèse selon laquelle les corps sont étendus dans un espace vide constituant le lieu de ces corps pour la raison qu'un tel espace possèderait des dimensions matérielles. Il prend l'exemple d'un cube de bois qui, lorsqu'il est plongé dans l'eau déplace un volume d'eau égal au sien, parce que les dimensions de ces deux corps ne peuvent coïncider. Or si les corps étaient immergés dans un espace vide, il faudrait que les dimensions des corps coïncident avec celles de cet espace ${ }^{31}$. La tradition aristotélicienne ultérieure fonde l'impénétrabilité non dans une qualité des corps, mais dans la possession de dimensions spatiales à la quantité qui est nécessairement corporelle ${ }^{32}$. L'originalité d'Ockham consiste à faire de l'impénétrabilité une propriété constitutive de la matière, non une conséquence d'un de ses accidents.

La théorie ockhamiste établit une implication réciproque de (1) avec (2) et (3) qui valide les principes d'harmonie méréologique pour les entités intrinsèquement étendues. Cependant plusieurs théories médiévales rejettent tout ou partie des liens logiques entre ces thèses à partir d'une conception réaliste de la quantité. Ainsi certains soutiennent la possibilité qu'une entité méréologiquement simple existe tout entière dans plusieurs corps à la fois. C'est le cas au sein de la tradition thomiste à propos de la matière première

31. Aristote, Physique IV, 1, 209a5-7 ; cf. E. Grant, op. cit., pp. 5-6.

32. Averroès, Commentaire sur la physique, IV, text. 76, cité in Anneliese Maier, art. cit. p. 147: "Impossibilitas penetrationis corporum accidit eis ex suis terminis mathematicis, non ex suis accidentibus sensibilibus "; Gilles de Rome, Comm. In Phys. IV, text. 76, Venise, 1502, f. 83ra, cité in Anneliese Maier, ibid., p. 149 : « Per se enim est quod dimensiones non compatiuntur secum dimensiones alias. " 
qui existe idem numero dans plusieurs choses à la fois ${ }^{33}$. Cette théorie de la matière première est compatible avec son caractère multilocalisé aussi bien qu'avec une forme d'extension ou de diffusion spatiale sans que l'on puisse proprement parler de partes extra partes. La théorie thomiste de la matière première parait entrainer la possibilité d'une extension sans parties, et donc comme un rejet de l'implication de (2) par (1).

Il existe une position symétriquement opposée, selon laquelle la matière possède intrinsèquement une structure méréologique complexe, autrement dit possède une diversité de parties intégrales, sans que ces parties occupent nécessairement une étendue spatiale déterminée, au point de pouvoir même être inétendues. Ainsi, cette position consiste à soutenir (2') et refuser (2") et donc (1). R. Pasnau la nomme "Extensionless Parts View ». Elle est notamment exposée et défendue par les Conimbricenses, en réponse à un argument établissant que la matière possède intrinsèquement des parties étendues parce qu'à chaque partie de l'étendue spatiale occupée par une substance matérielle correspond une partie de la matière :

La matière en elle-même, et mettant à part la quantité, possède des parties substantielles, à partir desquelles elle est composée. Cependant elle ne les possède pas étendues et disposées en ordre, et l'une en dehors de l'autre (unam extra aliam), sinon par l'intervention de la quantité, dont la fonction est d'expliquer et développer ces parties, qui seraient autrement implicites et confuses. [...] Il faut dire qu'aux parties de la quantité répondent les parties entitatives de la matière, réellement (re ipsa) distinctes tant les unes des autres que de la matière, mais il faut nier qu'elles soient étendues (extendi) sans l'aide de la quantité (In octo libros Ar. Physicorum, I, cap. II, q. 2, art. 3, Lyon, 1602, p. 116F-117A) ${ }^{34}$.

\section{La distinction cruciale est entre une multitudo extensiva et une multitudo} entitativa. La pluralité "entitative " de parties appartient intrinsèquement à la matière, mais l'ajout de la quantité est requis afin que cette pluralité forme une multitudo extensiva, pourvue d'une structure partes extra partes. L'acceptation de complexes inétendus est le symétrique inversé de l'acceptation d'étendus simples. Cette doctrine assigne à la quantité la fonction de constituer une extension spatiale de la matière, extension comprise comme la diffusion d'une structure méréologiquement complexe antérieure dans une extériorité spatiale.

33. Thomas d'Aquin, De principiis naturae, $\$ 2$, éd. Léonine, t. XLIII, Rome, 1976, p. $41 \mathrm{~b}$ : « Materia prima dicitur una numero in omnibus »; Jean Capreolus, In II Sent., d.18, q.1 ; Soncinas, Quaestiones metaphysicales, V, q.19, Lyon, 1576, p. 69b : "Sublata quantitate materia non habet partes [...] ratio partis non convenit eis, nisi per quantitatem. " Selon Soncinas, il est contradictoire de supposer que la matière subsiste réellement sans quantité (q. 22, p. 74ab). R. Pasnau (op. cit., p. 56) remarque qu'au XVI ${ }^{\mathrm{e}}$ siècle, Gabriel Vázquez défend une thèse explicitement holenmérienne à propos de la matière première et des substances matérielles considérées indépendamment de la quantité (In summam theologiae, III, disp. 187, cap. 2, n. 10 ; disp. 190, cap. 3, n. 27).

34. La question dans laquelle s'insère la discussion de cette thèse porte le titre: Sitne materia prima ex se dividua et extensa, an non ? Les Conimbricenses attribuent cette position à Duns Scot (Ord. II, d. 2, q. 4), Jean de Bassoles (Sent. IV, d.12, q.1, a.1) et Jean Mair. 
D'autres auteurs assignent un rôle différent à la quantité en distinguant entre les formes mêmes d'extension, à l'instar de Suárez. Ce dernier distingue ainsi entre extensio entitativa, extensio localis et extensio quantitatis ${ }^{35}$. La première correspond à la multiplicité des parties admise par les conimbricenses et chaque substance matérielle comme chaque portion de matière la possède intrinsèquement, indépendamment de la quantité. L'adjonction de la quantité est ce qui produit la seconde extension, en vertu de laquelle la chose occupe un lieu (ou encore possède une localisation circonscriptive) ${ }^{36}$. Mais cette extension ne désigne pas simplement la diffusion ni la possession de dimensions mesurables, contrairement à ce que laisse entendre la thèse des Conimbricenses. Selon Suárez, la quantité est la propriété en vertu de laquelle les corps molis corporea, propriété qu'on ne remarque que par son effet observable : la disposition de la res quanta à l'impénétrabilité, à exclure tout autre corps à occuper le même lieu (DM, disp. XL, sec. 4, n. 16-17).

La spécificité du réalisme suarézien de la quantité apparaît clairement dans la discussion d'une objection contre les théories qui font de la quantité le principe de l'extension spatiale des choses matérielles. L'objection part de l'hypothèse que la puissance divine peut conserver une matière étendue dans le lieu ou l'espace sans quantité et que l'étendue ne peut être une conséquence logique de la quantité (ibid., n.18).

Suárez commence par écarter trois réponses à cette objection. La première est la position thomiste qui rejette la situation envisagée comme une impossibilité37. Suárez exclut cette théorie qui ne respecte pas le principe de la nécessaire localisation de toute chose (ibid. n.19). La seconde réponse écartée consiste à soutenir que, dans l'hypothèse, la matière dépourvue de quantité serait réduite à un lieu dépourvu d'étendue, un poin ${ }^{38}$. La troisième solution est particulièrement intéressante : elle consiste à attribuer à la matière considérée indépendamment de la quantité une localisation dans une étendue divisible selon le mode d'une présence holenmérienne. La matière dépourvue de quantité serait alors un étendu simple ${ }^{39}$. Suárez pense qu'il s'agit d'une possibilité métaphysique qui requiert un miracle pour être actuelle, miracle qui se produit pour le corps du Christ dans l'Eucharistie. Néanmoins il rejette cette solution en ce qu'elle efface la différence essentielle entre les substances spirituelles, pour lesquelles la présence holenmérienne est naturelle, et les substances matérielles (ibid., n. 22).

35. Francisco Suárez, DM, disp. XL, sec. 4, n.15.

36. DM disp. LI, sec. 1, n.13; sec. 2 , n. 3 .

37. Cf. Cajetan, In Sum. theol., I, 52, 1, X, cité par Robert Pasnau, op. cit., p. 295.

38. C'est l'hypothèse envisagée par Pedro da Fonseca, In Metaph. V, 13, 2, 3, cité par Robert Pasnau, ibid.

39. Ibid., n. 21 : "Illam substantiam posse collocari... in spatio divisibili, ita tamen ut tota esset in qualibet parte ejus, quia, cum extensionem non haberet, non posset esse tota in toto et pars in parte. » Cette opinion est attribuée à Fonseca. C'est celle que défend Gabriel Vázquez et, au XIV siècle, Gauthier Burley qui soutient dans son traité De formis, que si la quantité était détruite une substance matérielle aurait le même mode d'être qu'une âme ; cf. Anneliese Maier, art. cit., pp. 216-217. 
C'est pourquoi il faut conclure, selon Suárez, que, dans l'hypothèse d'une destruction de la quantité, il n'y aurait aucun changement au point de vue des relations de localisation d'une substance matérielle $e^{40}$ :

Si donc la quantité était enlevée et la substance conservée et qu'il n’y avait nul autre changement de lieu dans la substance, celle-ci persisterait avec la même présence substantielle, et avec la même distance ou proximité au centre et aux pôles du monde ; et ainsi elle persisterait en étant présente tout entière au même espace et par parties (per partes) aux parties du même espace (DM disp. XL, sec. 4, n. 25 ; $c f$. sec. 2, n. 21).

La matière est pourvue donc d'une structure complexe naturellement congruente à la structure des lieux qu'elle occupe. C'est ici que se situe la différence avec la thèse, adoptée notamment par les Conimbricenses, selon laquelle "constituer une partie de la substance hors d'une autre partie est l'effet formel de la quantité " (ibid., n. 27).

Il y a en effet trois manières de penser l'extériorité des parties et une seule dépend de la quantité. La première est une extériorité "entitative ", synonyme d'une distinction réelle, sans connotation spatiale. C'est l'extériorité de la matière et de la forme.

La seconde est l'extériorité spatiale qui, lorsqu'elle est comprise comme une extériorité de localisation, paraît apparenter la substance matérielle non quantifiée à une substance immatérielle. Il y a toutefois une différence entre les deux. Une âme ou un ange n'est pas étendue par parties, mais existe tout entière dans le lieu qu'elle occupe et tout entière dans chacune de ses parties. Elle possède une existence holenmérienne dans ce que les scolastiques appellent un ubi definitivum. Inversement, la substance matérielle non quantifiée est étendue dans un lieu partes extra partes et possède ainsi un lieu circonscriptif, ubi circumscriptivum ${ }^{41}$.

Enfin, la dernière forme d'extériorité est l'extériorité quantitative, ainsi expliquée par Suárez : "Une partie est dite en dehors d'une autre par la répugnance naturelle à être à l'intérieur "(idem). Ainsi la quantité est la propriété en vertu de laquelle deux substances matérielles sont impénétrables ${ }^{42}$. Inversement, la structure partes extra partes n'implique pas d'elle-même une impénétrabilité. Les analyses précédentes permettent d'établir que Suárez soutient trois thèses distinctes :

- Une substance matérielle quantifiée est étendue partes extra partes et impénétrable.

- Une substance matérielle sans la quantité est étendue partes extra partes et pénétrable.

40. Rappelons que le fait d'être ubi est pour chaque chose un mode intrinsèque de celle-ci, indépendant de ses accidents, donc en particulier de sa quantité.

41. Cf. Thomas d'Aquin, Summa theologiae, I, 52, 2, in corp.; Guillaume d'Ockham, Quodl. IV, q. 21, OTh IX, p. 400 sq. ; Francisco Suárez, DM disp. LI, sec. 6, n. 2-6.

42. DM disp. XL, sec. 2, n. 20 ; sec. 4, n. 15-17 ; cf. Robert Pasnau, op. cit., pp. 312-314. 
- Une substance immatérielle n'est pas étendue partes extra partes, mais selon le mode holenmérien, et elle est pénétrable.

Ainsi, l'étendue n'implique pas nécessairement la possession d'une structure méréologique, comme le montre le cas des âmes et des substances immatérielles; une étendue méréologiquement complexe n’implique pas nécessairement à son tour l'impénétrabilité, à condition d'admettre la possibilité d'une matière dépourvue de quantité.

\section{L'argument cartésien pour l'impénétrabilité et sa critique par More}

Il est possible de se pencher de nouveau sur la discussion entre Descartes et More à la lumière des différentes possibilités théoriques offertes par les scolastiques. Descartes, on l'a vu, soutient que la structure partes extra partes implique l'impénétrabilité. More au contraire soutient que dans le cas particulier de l'espace, l'implication n'est pas vérifiée.

Dans la lettre du 15 avril 1649, Descartes présente un argument fondé sur une expérience de pensée pour établir sa thèse :

On ne peut pas entendre (intelligi) non plus qu'une partie d'une chose étendue en pénètre une autre qui lui soit égale, sans que par là même on n'entende (intelligatur) qu’une partie intermédiaire de son étendue ne soit supprimée ou annihilée. Or ce qui est annihilé ne peut pénétrer une autre chose ; et on démontre ainsi, selon moi, que l'impénétrabilité appartient à l'essence de l'étendue, et non à l'essence d'aucune autre chose (AT V, 342).

La situation envisagée par Descartes est la suivante ${ }^{43}$ : un corps sphérique d'un mètre cube est mu de telle façon qu'il coïncide avec un autre corps sphérique d'un mètre cube. Avant le mouvement, il y avait deux mètres cubes d'étendue, après, il n'y a en qu'un seul. Donc la moitié de l'étendue ayant disparu, on ne peut dire qu'une portion en a pénétré une autre, car "ce qui est annihilé ne peut pénétrer autre chose ». Comme l'écrit Dan Garber : "Si le corps est une chose étendue [...], alors enlevez l'étendue et vous enlevez le corps. Mais si deux corps pouvaient se pénétrer, le volume total, et donc une partie du corps lui-même, serait éliminé. Mais si l'un ou l'autre des deux corps est éliminé par cette interpénétration, alors il n’y a pas d'interpénétration réelle " (Garber 1992, p. 147). L'argument, " simple et ingénieux » selon D. Garber (ibid.), paraît en effet imparable si l'on imagine deux corps, mais l'argument est censé convaincre More que deux portions d'étendue en général sont impénétrables. Or c'est ce qui ne convainc nullement le philosophe anglais comme l'indique sa réponse dans la lettre du 29 juillet 1649 :

43. Voir Daniel Garber, op. cit., pp. 147-148; Jonathan Bennett Learning from Six Philosophers, Oxford, Clarendon, 2001, t. I, pp. 31-35; Robert Pasnau, art. cit. et op. cit., pp. 318-319. 
[D]ans une substance étendue il peut y avoir des parties les unes en dehors des autres sans aucune antitypie, ou résistance mutuelle, et ainsi périt la tangibilité proprement dite. En outre, une étendue avec une substance (extensionem simul cum substantia) peut être repliée (replicari) sur une étendue et une substance restante et celle-ci n'est pas davantage perdue que la partie de la substance qui est repliée (retrahitur) dans l'autre, et par là tombe l'impénétrabilité. Cela, j'affirme le concevoir clairement et distinctement en mon esprit (AT V, 378).

More oppose ainsi à l'inconcevabilité sur laquelle s'appuie Descartes sa capacité à concevoir le scénario en question. L'étendue est pénétrable parce qu'il parvient à le concevoir clairement et distinctement. Certes l'étendue est immobile et nulle partie ne peut effectivement être mue pour coïncider avec une autre ${ }^{44}$. S'il peut donc concevoir un tel scénario, c'est parce qu'il distingue l'étendue des substances qui l'occupent. Or n'importe quelle substance peut coïncider avec l'étendue spatiale qu'elle occupe. Davantage, deux substances spirituelles peuvent coïncider l'une avec l'autre.

La réaction de More conforte le jugement de J. Bennett qui estime que l'argument de Descartes ne saurait convertir quiconque à sa thèse. L'argument présuppose en effet que la fusion d'un corps de volume $V$ et d'un autre corps de volume $V^{\prime}$ doit occuper un volume $\left(V+V^{\prime}\right)^{45}$. Or c'est précisément cela qui est en question. More lui oppose que deux substances spirituelles pourvues chacune d'une certaine étendue peuvent coexister dans un même lieu, de telle sorte qu'elles occupent une étendue moindre que leurs deux étendues séparées.

Cependant, l'argument de Descartes ne repose pas seulement sur le concept d'étendue, mais sur la structure partes extra partes de la res extensa et la conservation divine de celle-ci. Pour établir ce point, il convient de rapprocher l'argument de concevabilité de la correspondance avec More des développements sur la condensation et la raréfaction dans les Principes. L'étendue d'un corps est invariable dans la mesure où le corps lui-même est identifié à une portion d'étendue. L'erreur qui consiste à croire en la possibilité d'une raréfaction et condensation absolues provient soit de la distinction entre la substance corporelle et la quantité, à la manière des Conimbricenses, soit de la distinction ultérieure entre quantité et étendue à la manière de Suárez ${ }^{46}$. L'augmentation de la quantité ou de l'étendue ne peut se produire qu'à condition de rompre la conservation des corps :

Ac denique plane repugnat aliquid nova quantitate vel nova extensione augeri, quin simul etiam nova substantia extensa, hoc est, novum corpus ei accedat. Neque enim ullum additamentum extensionis vel quantitatis, sine additamento substantiae quae sit quanta et extensa, potest intelligi (PP II, art. 7, AT VIII-1, 44).

44. Cf. Lettre à Anne Conway, 5 mai 1651, éd. A. Gabbey, p. 389.

45. Jonathan Bennett, op. cit., t. I, p. 31, auquel s'oppose Robert Pasnau, art. cit., p. 302. J. Reid (cf. op. cit., pp. 67-71) défend une position comparable.

46. PP II, 5, AT VIII-1, 42 : «Sintque etiam nonnulli adeo subtiles, ut substantiam corporis $\mathrm{ab}$ ejusdem quantitate, atque quantitatem ab extensione distinguant. » 
Une étendue plus grande ne peut être occupée à un moment qu'à un moment antérieur, que si une nouvelle substance est créée, comme l'avait déjà soutenu Ockham. Réciproquement, bien que Descartes ne l'explicite pas ici, toute diminution de l'étendue tridimensionnelle implique la destruction d'une substance. Or Dieu, en vertu de son immutabilité, conserve à chaque instant autant de matière qu'il en a créé initialement ${ }^{47}$. Le principe de conservation de la quantité de matière ou de substance étendue fonde l'impossibilité d'une raréfaction et d'une condensation absolue. L'impénétrabilité n'est donc pas une propriété déductible d'une analyse conceptuelle de l'étendue. Il faut y ajouter la considération des lois de la nature et du principe de conservation divin de la quantité de matière ${ }^{48}$.

L'avantage de cette lecture est qu'elle permet d'éviter d'attribuer à Descartes une contradiction flagrante lorsqu'il soutient par ailleurs que l'âme est co-localisée avec le corps et notamment qu'il est possible de la concevoir comme co-étendue avec ce dernier. Or dans la lettre à l'Hyperaspistes il écrit :

Quomodo mens corpori extenso coextendatur, etsi non habeat ullam veram extensionem, hoc est ullam per quam locum occupet, atque aliud quid ab eo excludat, iam supra exemplo gravitatis sumptae pro qualitate reali explicui (AT III, 434) ${ }^{49}$.

L'extension que Descartes attribue à la pensée ou à l'âme est une extension qui n'empêche pas la pénétration. C'est là un point capital, sans quoi l'âme ne pourrait pas être réellement unie au $\operatorname{corps}^{50}$. Mais quelle est la nature de cette étendue qui n'est pas impénétrable?

Le renvoi à l'analyse bien connue de la pesanteur conçue comme qualité réelle dans les Sixièmes réponses permet de répondre à cette question ${ }^{51}$. Parce que la pesanteur agit dans le corps grave tout entier, nous imaginons qu'elle est répandue dans ce corps entier (per totum corpus... sparsam esse imaginarer).

47. PP II, 36, AT VIII-1, 62, 1. 2-4 : « Jamque totam istam materiam conservet eodem plane modo eademque ratione qua prius creavit."

48. Nous suivons en cela l'analyse défendue par Robert Pasnau, op. cit., pp. 319-322. Voir en particulier p. 322 : «Les corps résistent à la co-localisation non en vertu d'une caractéristique intrinsèque qui empêcherait causalement que la pénétration se produise, mais parce que les lois de Dieu les en empêchent. "

49. Cf. Lettre à Elisabeth, 28 juin 1643, AT III, 694 : "L'extension de cette matière est d'autre nature que l'extension de cette pensée, en ce que la première est déterminée à certain lieu, duquel elle exclut toute autre extension de corps, ce que ne fait pas la deuxième. "

50. Cela suppose une interprétation de la thèse de Descartes comme n'étant pas nullibiste. Voir sur cette notion l'article de David Leech dans ce volume. La distinction entre l'étendue de substance et l'étendue de puissance dans la correspondance avec More paraît ranger Descartes dans le camp nullibiste. On peut néanmoins proposer une interprétation de Pétendue de puissance comme étendue intrinsèque, mais non nécessaire, de la substance pensante. Voir sur ce point Marleen Rozemond, art. cit., ainsi que notre article Jean-Pascal Anfray, "L'étendue spatiale et temporelle des esprits : Descartes et le holenmérisme ", Revue philosophique de la France et de l'étranger, (à paraître) 2014/1.

51. Sixièmes réponses, AT VII, 441-42; Lettre à Elisabeth, 21 mai 1643, AT III, 667 ; Lettre à Arnauld, 29 juillet 1648, AT V, 222-23. 
En outre, l'étendue corporelle est impénétrable et ne peut donc se contracter absolument, ce que peut la pesanteur capable d'exercer son action en un point mathématique (eandem in punctum Mathematicum contrahi posse judicabam). Il ajoute enfin que la pesanteur peut agir sur n'importe quelle partie du corps grave tout entier, ce quatteste la tension que subirait un fil auquel serait suspendu le corps, tension proportionnelle au poids du corps entier et non de la partie fixée à l'extrémité du fil. Et Descartes conclut que c'est ainsi que nous devons penser l'étendue de l'âme :

Nec sane jam mentem alia ratione corpori coextensam, totamque in toto, et totam in qualibet ejus parte esse intelligo (AT VII, 442).

Il précise enfin que la pesanteur est divisible et mesurable, à la différence de l'âme. L'étendue de l'âme est ainsi une étendue indivisible et nonmesurable qui relève d'une présence holenmérienne. Comme on l'a vu dans l'introduction, la caractéristique d'une existence holenmérienne est de ne pas impliquer de correspondance entre la structure méréologique de l'entité localisée, ici l'âme, et celle de l'entité avec laquelle elle est colocalisée. L'étendue de l'âme selon Descartes n'est donc pas celle d'une entité partes extra partes qui ne serait pas impénétrable comme chez Suárez. On peut douter qu'il s'agisse d'une étendue même dans un sens atténué, dans la mesure où elle n'est pas mesurable. Il conviendrait plutôt de parler d'une multi-localisation de l'âme dans le corps. En retour, l'analyse de l' "étendue de la pensée " chez Descartes confirme bien que la structure partes extra partes implique en effet l'impénétrabilité, moyennant la conservation divine.

\section{Evolution chez More du rapport entre étendue spirituelle et structure partes extra partes}

Si l'analyse des différentes positions scolastiques sur le rapport entre matière, étendue et structure partes extra partes contribue à mieux saisir les implications de la position de Descartes et de l'un de ses arguments les plus discutés dans la correspondance, elle permet également d'éclairer deux concepts de More élaborés après la correspondance avec Descartes pour penser l'étendue spirituelle : le concept de spissitude essentielle et celui d'inscerpibilité.

More rejette la conclusion de l'argument de concevabilité dans la correspondance. Par ailleurs, il affirme que l'étendue spirituelle est variable si bien qu'une même substance peut occuper une étendue spatiale plus ou moins grande à différents moments du temps, et en effet elle peut réellement se dilater ou se contracter (AT V, 378-79). L'interdit cartésien d'une condensation et dilatation absolue n'a ainsi qu'une portée relative, restreinte aux phénomènes naturels. Dans The Immortality of the Soul, More articule 
directement l'affirmation de la pénétrabilité à celle de la variabilité de l'étendue via la notion de spissitude essentielle des esprits :

Car la substance n'est pas davantage perdue dans ce cas que lorsqu'une corde est doublée ou redoublée, ou qu'un morceau de cire est réduit d'une figure allongée en une figure ronde : la dimension de la longueur est en quelque façon perdue, mais sans que ce soit au détriment de la substance de la cire. Pareillement, lorsqu'une partie d'une substance étendue en rencontre une autre, quelque chose est perdu à la fois de la longueur, de la largeur et de la profondeur, et pourtant toute la substance subsiste toujours [...]. Et de même ce qui a été perdu en longueur a été gagné en largeur ou profondeur auparavant; de même ce qui a été perdu dans toutes les dimensions ou deux quelconques d'entre elles est préservé dans la spissitude essentielle : c'est ainsi que j'appellerai ce mode ou cette propriété de la substance d'être capable de recevoir une partie d'elle-même dans une autre (The Immortality of the Soul, I, chap. II, $\$ 11$, éd. A. Jacob, p. 28).

L'interprétation exacte de la nature de la spissitude n'est pas évidente. Le texte précédent semble indiquer clairement qu'il s'agit d'une quatrième dimension qui permet de garantir une forme de conservation des dimensions : ce qui est gagné ou perdu dans les dimensions spatiales est corrélativement perdu ou gagné par la spissitude essentielle de la substance. Cependant, dans d'autres passages, la notion de spissitude désigne plutôt quelque chose d'analogue à la densité dans les corps ${ }^{52}$. Nous voudrions suggérer ici une autre piste, qui nous rattache aux discussions scolastiques sur la quantité. On se souvient que selon la théorie des parties sans extension, un corps a une complexité méréologique intrinsèque et c'est l'accident de la quantité qui confère une relation d'extériorité et de distance spatiale à ces parties (cf. supra p. 49). La théorie de la spissitude essentielle proposée par More ressemble à première vue à une transposition dans le cas des esprits de cette théorie. En effet, les substances spirituelles sont essentiellement complexes, mais ces parties ne sont pas nécessairement extérieures les unes aux autres. La spissitude moréenne se distingue des théories comme celles des Conimbricenses en ce qu'il exclut la possibilité d'une substance dépourvue de toute étendue. Il soutient cela dès la lettre à Descartes du 5 mars 1649, en s'appuyant sur l'autorité de Jules César Scaliger :

Mais il arrive ici à mon imagination ce que Jules Scaliger écrit quelque part de la dilatation et de la contraction des anges, à savoir qu'ils ne peuvent s'étendre à l'infini, ni se rétrécir au néant d'un point $(\mathrm{AT} \mathrm{V}, 304)^{53}$.

52. Voir Jasper Reid, op. cit., pp. 202-205.

53. $C f$. Jules César Scaliger, Exotericarum exercitationum liber quintus de subtilitate, ad Hieronimum Cardanum, Paris, 1557, exercit. 309, 6, p. 463 : "Contrahi vero, et diffundi Angelos asserta sibi magnitudine, sed certa, finitaque, verum est. [...] Non enim in infinitum sese promoverit, neque ad puncti oudenotêta sese contraxerit. "Sur l'influence de Scaliger sur la pensée de More, voir I. Agostini, art. cit. 
La spissitude implique une forme de résistance à la contraction qui devient absolue au-delà d'un certain seuil hypothétique. L'interprétation de la spissitude en termes méréologiques est utile ici : toute substance possède bien une structure spatiale minimale, mais celle-ci est susceptible de variations jusqu'à une certaine limite, l'étendue occupée par un esprit étant nécessairement finie.

La seconde notion est celle d'indiscerpibilité. Un être est discerpible s'il peut être divisé en parties distinctes ${ }^{54}$. L'étendue spirituelle, ainsi que l'étendue spatiale sont indiscerpibles. Nous avons vu qu'à l'époque de la correspondance, More soutenait que l'étendue divine et l'étendue spirituelle étaient des étendues véritables, mais qu'il hésitait sur le fait de savoir si cette étendue était une étendue pars extra partem. Ou plus précisément, il est manifeste que, dans la correspondance, il admet une forme de mesurabilité des substances immatérielles, en même temps qu'il recourt à un vocabulaire holenmérien pour penser leur localisation. Les esprits sont étendus au sens où ils sont localisés dans tel et tel lieu de l'espace, ces lieux étant eux-mêmes distants. Néanmoins, il s'agit d'une étendue sans partition, autrement dit il s'agit de les considérer comme des étendus simples ${ }^{55}$. Or More maintient cette thèse dans ses écrits ultérieurs, alors qu'il rejette très nettement le holenmérisme à partir de 1659 avec la publication de The Immortality of the Sou ${ }^{56}$. Dès lors, il lui faut pouvoir penser une étendue simple et indivisible qui ne se confonde pas avec l'existence holenmérienne. Mais en niant que l'âme soit tout entière présente dans telle partie de l'espace ou du corps, More n'a d'autre issue que de conclure que l'âme est étendue partie par partie, que, par exemple, une des parties de son étendue coïncide avec celle du pied et une autre avec celle de la tête. Ainsi l'étendue spirituelle se voit attribuer la structure partes extra partes que More accordait à l'espace dès l'époque des lettres à Descartes. Celle-ci revient à accorder à ces deux étendues le statut de l'étendue matérielle

54. The Immortality of the Soul (1659), I, chap. II, $\$ 10$, éd. A. Jacob, Dordrecht, Kluwer (Archives internationales d'histoire des idées 122) 1987, p. 27 : "I understand Discerpibility, gross tearing or cutting one part from another. " Les atomes non figurés sont également indiscerpibles, mais ils sont impénétrables. Voir. The Immortality of the Soul, préface, $\$ 3$, p. 6 : "Particles that have indeed real extension, but so little, that they cannot have less and be any thing at all, and therefore cannot be actually divided. Which minute extension if you will, you may call Essential »; Enhiridion metaphysicum, chap. XXVIII, $\$ 10$, OO II-1, 321. Sur l'indiscerpibilité, voir Jasper Reid, op. cit., pp. 188-194.

55. Voir Robert Pasnau, art. cit., pp. 304-309. Mais ce dernier est conduit à une difficulté tenant à l'absence de prise en compte de l'évolution de More sur cette question. Si l'étendue sans partition est bien la marque du non-physique et si, en 1649, More l'explique dans un schème holenmérien, il rejette toutefois le holenmérisme au moment où il introduit le concept d'indiscerpibilité.

56. The Immortality of the Soul I, chap. X, $\$ 8$, éd. A. Jacob p. 58 ; EM, chap. XXVII, $\$ 11-15$, OO II-1, 312-315. Dans la Preface General de la Collection de 1662, More développe la même série d'arguments contre le holenmérisme, en indiquant que l'Immortality of the Soul marque un tournant dans sa pensée : A Collection of Several Philosophical Writings, Pref., $\$ 12$, p. xii-xiii. Sur l'évolution de More et sa critique progressive du holenmérisme, voir Jasper Reid, op. cit., pp. 158-174. 
non quantifiée chez Suárez. Il s'agit d'une conséquence logique du rejet du holenmérisme, malgré certaines protestations contraires de More :

[...] [D]e ce qu'une chose est étendue, on imagine aussitôt qu'elle possède des parties les unes en dehors des autres (partes habere extra partes) et qu'elle n'est pas une unité par soi, mais le résultat d'une juxtaposition de parties. Alors que l'idée d'étendue précisément considérée en soi n'inclut rien de tel, mais seulement une amplitude tri-dimensionnelle ou solide ( $E M$ chap. XXVIII, $\$$ 9, OO II-1, 321 $)^{57}$.

À première vue, More oppose clairement l'étendue simple à la structure partes extra partes. L'indiscerpibilité semble bien devoir être comprise comme une étendue sans partition. Néanmoins on remarque que More oppose l'amplitude ou l'étendue spirituelle à une double affirmation : (i) la possession d'une structure partes extra partes et (ii) le fait que le tout étendu possède seulement une unité de composition. Cette unité de composition caractérise seulement certains touts, ceux dont les parties sont physiquement divisibles. Or certains passages indiquent clairement que les esprits possèdent des parties, bien qu'elles soient absolument inséparables : «No other Vinculum to hold the parts together but its own essence and existence ; whence it is of its own nature indiscerpible ${ }^{58}$." Les parties physiques sont celles qui peuvent subsister indépendamment des autres, conformément au critère cartésien de la distinction réelle. Mais selon More, l'unité de l'étendue spirituelle est telle qu'elle est absolument indissoluble, si bien que les parties qui peuvent y être distinguées notionnellement ne peuvent subsister par elle-même ${ }^{59}$. L'étendue indiscerpible est donc bien une étendue par partition, à condition d'admettre que ces parties ne peuvent subsister indépendamment de l'étendue totale ${ }^{60}$. On conçoit bien la position que More souhaite défendre. Il s'agit de répondre aux arguments des nullibistes et des matérialistes qui identifient l'étendue à une structure partes extra partes impliquant la divisibilité de la res extensa. More lui oppose l'idée d'une étendue par partition notionnelle compatible avec l'unité et la simplicité métaphysique de l'étendue spirituelle ${ }^{61}$. Mais il ne

57. EM, chap. XXVII, $₫ 12$, OO II-1, 313 : «Extensio Spirituum metaphysica nec magnitudinis nec partium est capax "; scholia $\$ 14$ : "Ens simplex [est] cujus substantiae amplitudo nullam habeat coagmentationem partium, sed una omnino sit simplexque substantia sine partibus physicis."

58. Divine Dialogues (1668), dial. I, $\$ 30$, cité in J. Reid, op. cit., p. 190.

59. Enchiridion metpahysicum, chap. XXVIII, \$ 10, OO II-1, 321 : "Rem vero extensam Logice vel intellectualiter dividi posse, cum tamen nullo pacto possit discerpi..."

60. Idem: "Et certe in Monade metaphysica, qualem Holenmeriani Mentem humanam esse fingunt, totumque interim corpus eodem tempore occupare et possidere, logica hic iterum fieri potest distributio, puta e subjectis, quatenus scilicet haec Monas metaphysica sive Holenmerianorum Anima, vel Caput vel Truncum vel Artus possidet. "

61. L'indiscerpibilité correspond d'assez près à la caractérisation par Suárez de la conception de l'espace comme contenant tridimensionnel qu'il attribue à Jean Philopon notamment ; $c f$. Francisco Suárez, DM disp. LI, sec.1, n. 10: «Forte intellexebatur esse divisibile non quia caret partibus, sed quia disiungi non possunt. " 
semble pas posséder d'argument pour éviter la conclusion selon laquelle les parties distinguées ainsi par la pensée sont également réellement divisibles. Dans le cas de l'étendue spatiale, il peut s'appuyer sur l'immutabilité absolue des parties qui interdit qu'elles soient séparées les unes des autres. Cette immutabilité peut s'appliquer à l'étendue divine, mais elle est incompatible avec la nature de l'étendue spirituelle qui est précisément mobile.

L'histoire de la notion d'étendue par partition ou encore de la possession d'une structure méréologique complexe, c'est-à-dire de l'étendue comme structure partes extra partes, reste encore à écrire. Nous avons jeté ici quelques jalons et avons insisté principalement sur le contexte scolastique. Il ne s'agit pas de prétendre que la théorie cartésienne de la res extensa se réduit in fine à la théorie d'Ockham ni en retour de voir dans les développements de la notion d'étendue spirituelle l'influence directe des discussions médiévales sur la quantité. Celles-ci dessinent toutefois, d'une façon inattendue, les contours de la discussion autour des liens conceptuels entre étendue et impénétrabilité.

Jean-Pascal ANFraY 